Eduard Kucera

\title{
Anatomical changes after hysteroscopic transcervical uterine septum resection-is there a place for hysterography?
}

Published online: 24 December 2004

(C) Springer-Verlag Berlin / Heidelberg 2004

\begin{abstract}
The anatomical changes of the uterine cavity after transcervical septum resection are reported. We measured the distance between the apices of the uterine horns and the extent of the uterine septum before and after the hysteroscopic resection of the septum. We calculated the index of the uterine cavity as a distance in millimeters between the uterine horns divided by the length of the uterine septum multiplied by 100 . The retrospective study was performed in 43 women with transcervical uterine septum resection treated between 1999-2002 by the Institute for the Care of Mother and Child of Prague and the Institute for Postgraduate Medical Education of Prague. Hysteroscopic resection of the uterine septum with evaluation of the surgical effect by hysterography was indicated pre- and postoperatively. For many years, hysteroscopic transcervical uterine septum resection has been a method of choice in the treatment of congenital uterine malformations. We operated on 43 women and achieved 27 intrauterine pregnancies $(62.8 \%)$, including 4 spontaneous abortions $(14.8 \%)$. The aim of our study was to evaluate the changes of the uterine cavity after resectoscopic surgery and find out if there is any correlation between residual septum and pregnancy outcome. The uterine cavity index was measured and calculated from hysterograms. Diagnostic hysterography was performed before and 2-3 months after surgery. The results showed significant changes of the calculated ratio before and after surgery. When the above-mentioned ratio is $10 \%$ or less, reproductive
\end{abstract}

This work was supported by the research grant CZ MZOL 34-000-000-1

\section{E. Kucera}

Institute for the Care of Mother and Child,

Institute for Postgraduate Medical Education,

Podolské nábřeži 157, 414700 Prague, Czech Republic

E-mail: ed_kuc@seznam.cz

Tel.: + 42-0296511827 failure is not expected. There is a higher incidence of spontaneous abortion when the ratio is more than $10 \%$. Therefore, especially in difficult cases of uterine septum resection (e.g., hypoplastic septate uterus, repeat pregnancy loss or failure after surgery), postoperative evaluation of the uterine cavity is feasible.

Keywords Infertility - Uterine septum · Hysterography · Hysteroscopy · Miscarriage ·

Uterine malformation

\section{Introduction}

The exact prevalence of septate uterus in the general population is still undetermined, but an estimate of $1 \%$ may be considered near the true figure [13]. The prevalence in women with repeated pregnancy loss is significantly higher $(3-4 \%)$. The reproductive outcome in women with septate uterus is characterized by an increased incidence of spontaneous abortion, premature birth and abnormal fetal presentations [8, 9, 10, 15]. Physiological pregnancy and term delivery are the optimal clinical outcomes after hysteroscopic transcervical resection of uterine septum. Different methods are used for evaluation of the uterine cavity. TVS, sonohysterography, 3-D ultrasound or diagnostic hysteroscopy is the methods of choice $[1,4,7,10,11]$. Less attention is paid to the postoperative evaluation of the uterine cavity.

Hysterography is able to evaluate the whole uterine cavity, as well as the length of the uterine septum and the distance of the apices of the uterine horns (Fig. 1) $[12,14]$. We measured the distance between the apices of the uterine horns and the extent of the uterine septum before and after the hysteroscopic resection of the septum. We calculated the uterine cavity ratio as a distance in millimeters between the uterine horns, divided by the length of the uterine septum, and multiplied by 100 . This calculated ratio in percent gives us a 


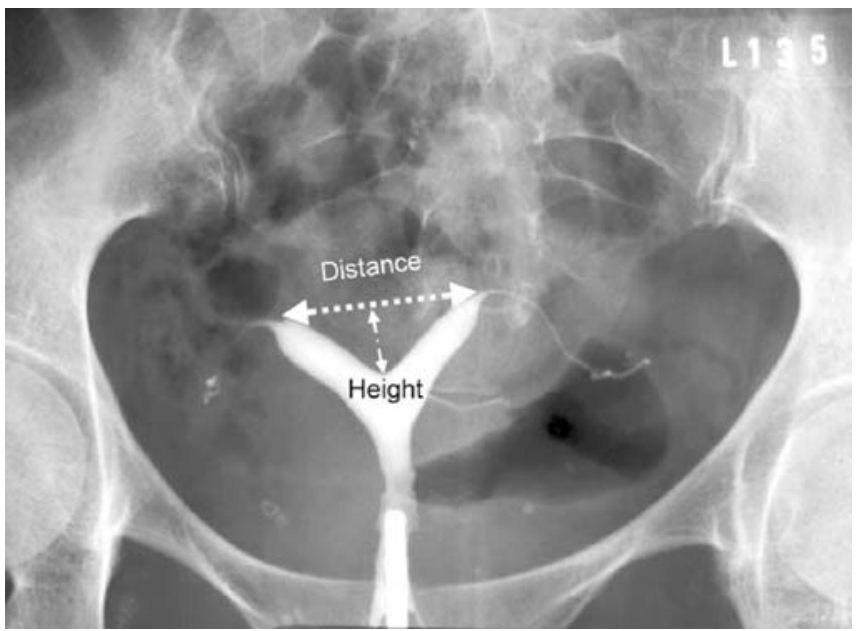

Fig. 1 Hysterographic preoperative diagrammatic representation of uterine cavity ratio

good understanding of the anatomical changes before and after hysteroscopic septum resection (Fig. 2). The unsatisfactory postoperative results with persistent or residual uterine septum can be diagnosed by this method. The postoperative counseling and future management of the infertile couple can be established according to the calculated results.

\section{Materials and methods}

In an analytical, non-randomized study, we tried to ascertain the anatomical changes of the uterine cavity before and after hysteroscopic uterine septum resection in group I. Hysterography was performed before and 2-

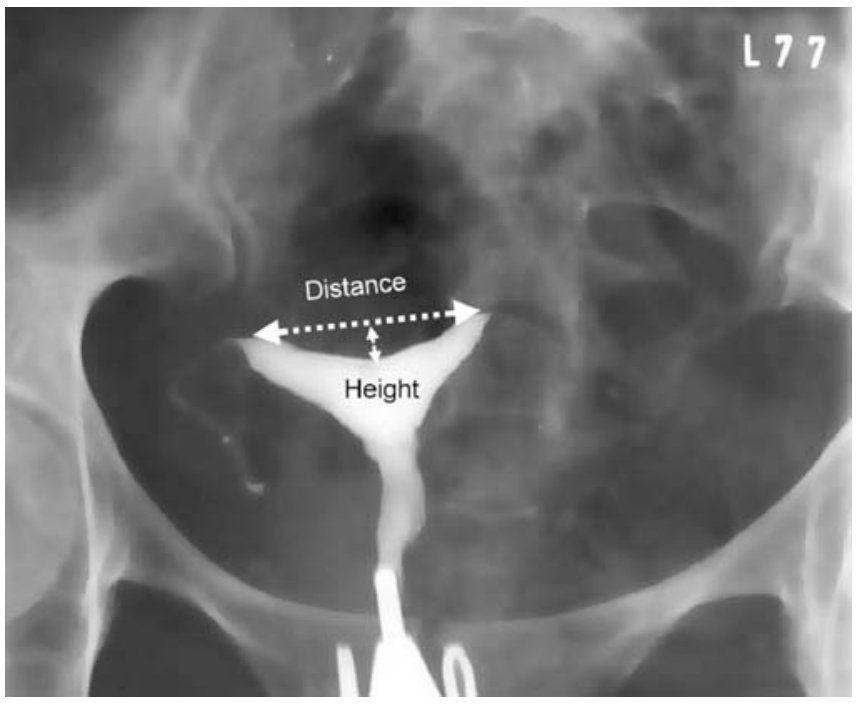

Fig. 2 Postoperative hysterographic representation of uterine cavity ratio in the same patient
3 month after surgery in the early follicular phase of the menstruation cycle. For hysterographic examination, we used Telebrix N (Byk Gulden, Germany ). Radiograms were taken in the A-P directions. With the radiograms from hysterography, a ratio was derived in millimeters between the height of the fundic impression (septum) indenting the uterine cavity and the distance between the apices of the uterine horns. The ratio was calculated in percent. There is evidence in the literature to show that when the ratio is $10 \%$ or less, reproductive failure is not expected (e.g., in the arcuate uterus, the ratio is less than $10 \%)[12,14]$.

Hysteroscopic resection of the uterine septum was performed in the early follicular phase under general anesthesia. The 8-mm continuous-flow resectoscope (Storz, Tutlingen, Germany) with a monopolar knifeshaped coagulating electrode at a power setting of 80 $120 \mathrm{~W}$ was used. We used Purisol (Fresenius-Kabi, Austria) for the uterine cavity distension. We did not use any endometrial suppression therapy before surgery. For laparoscopic evaluation, the standardized diagnostic procedure was used for the hysteroscopic procedure. According to the aim of our study, we evaluated the pregnancy outcome after surgery. Statistical analysis was performed using the $\mathrm{X}^{2}$-test.

\section{Results}

Forty-three infertile women who underwent hysteroscopic septum resection in 1999-2002 were studied. The average age of the patients was 31 years (21-42 years). Thirty-one $(68.9 \%)$ women were subjected to laparoscopic control for definitive uterine morphology evaluation for the hysteroscopic transcervical septum resection. The uterine morphology in 12 women $(31.9 \%)$ was already known from their medical history. The principal method used to identify the failure of septal resorption was hysterography $(n=28)$ (Figs. 3, 4,

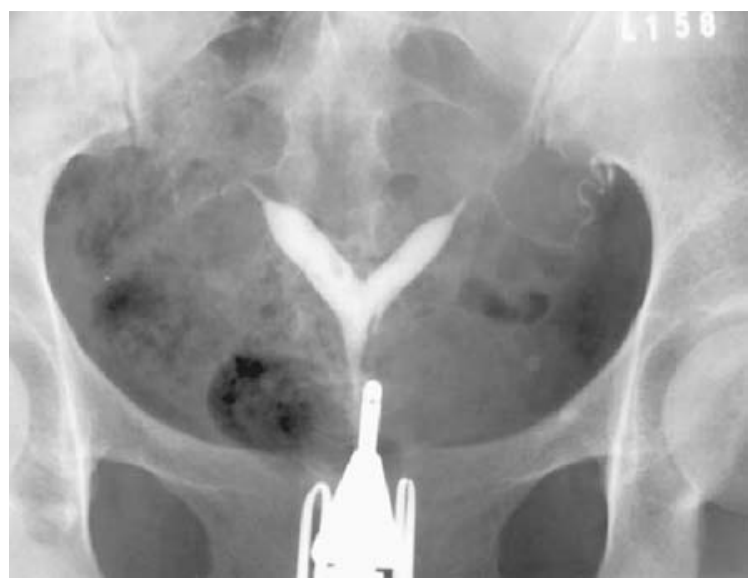

Fig. 3 Hysterogram in the same patient with two-step septum resection. Ratio $57 \%$ 


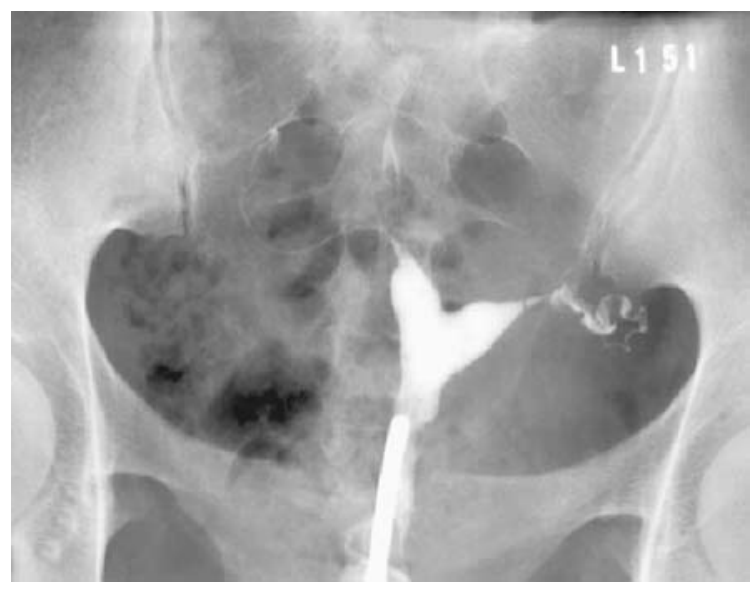

Fig. 4 Hysterogram in the same patient with two-step septum resection. Ratio $36 \%$

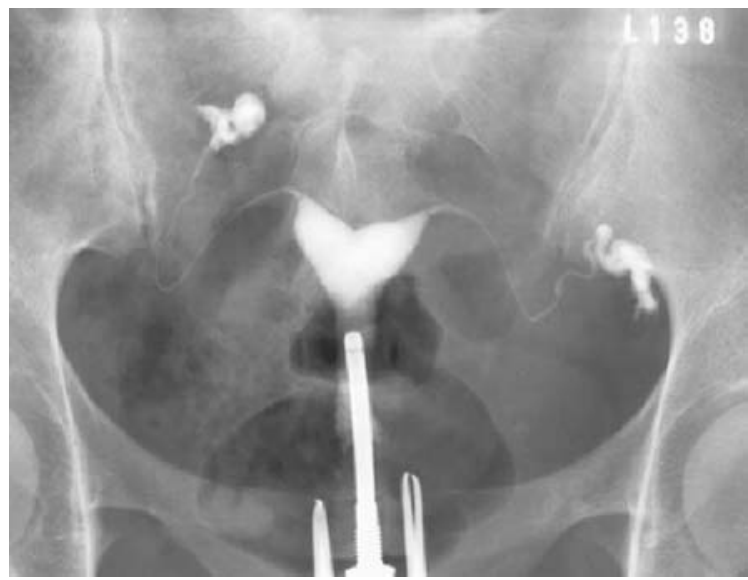

Fig. 5 Hysterogram in the same patient with two-step septum resection. Ratio $<10 \%$

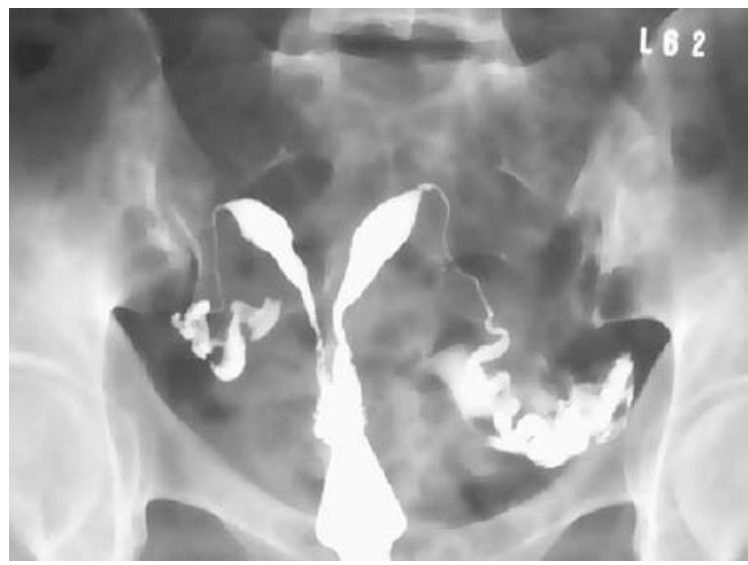

Fig. 6 Difficult case of uterine septum resection - complete septate uterus with both uterine horns connecting to single cervical os. Preoperative ratio $100 \%$

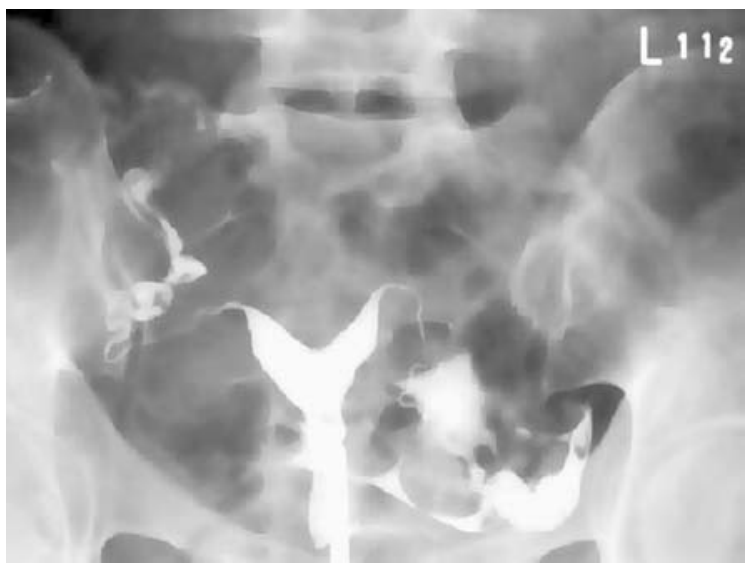

Fig. 7 Difficult case of uterine septum resection - complete septate uterus with both uterine horns connecting to single cervical os. Postoperative ratio $33 \%$

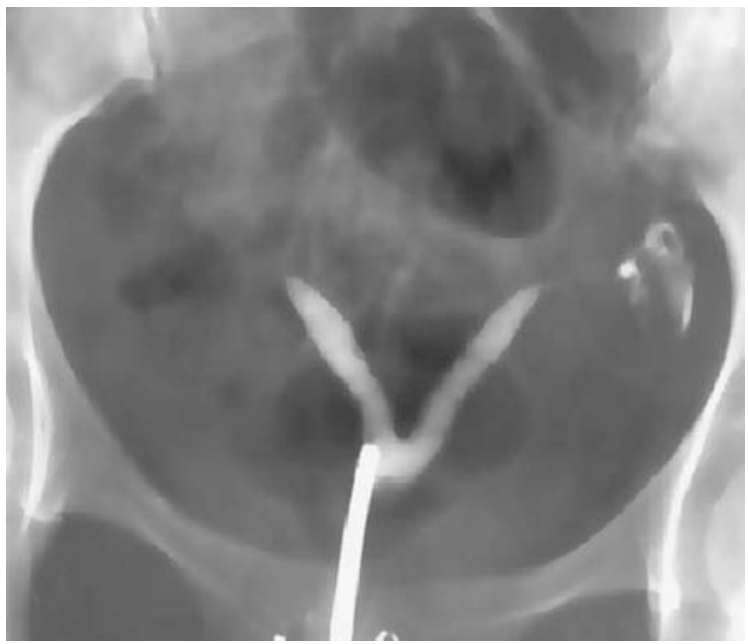

Fig. 8 Reconstruction of the uterine cavity after previous insufficient Strassman metroplasty. Uterine septum ratio $72 \%$

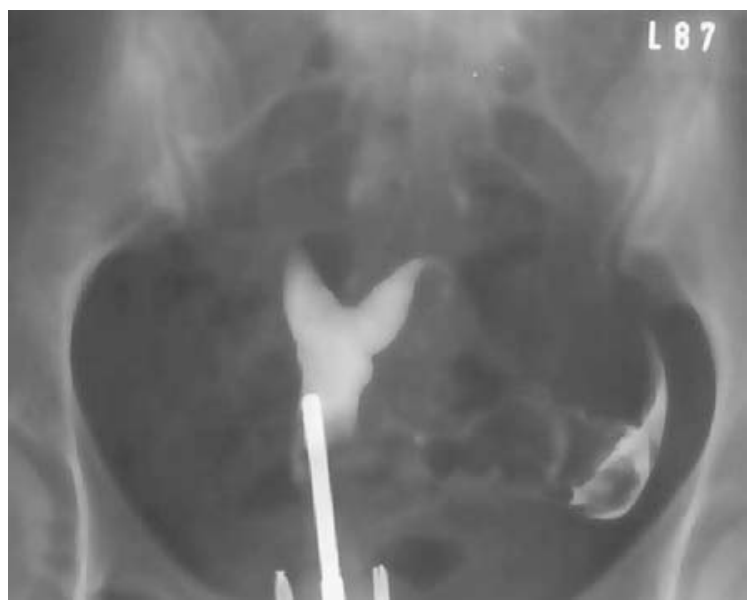

Fig. 9 Reconstruction of the uterine cavity after previous insufficient Strassman metroplasty. Persistent septum after Strassman metroplasty ratio $45 \%$ 


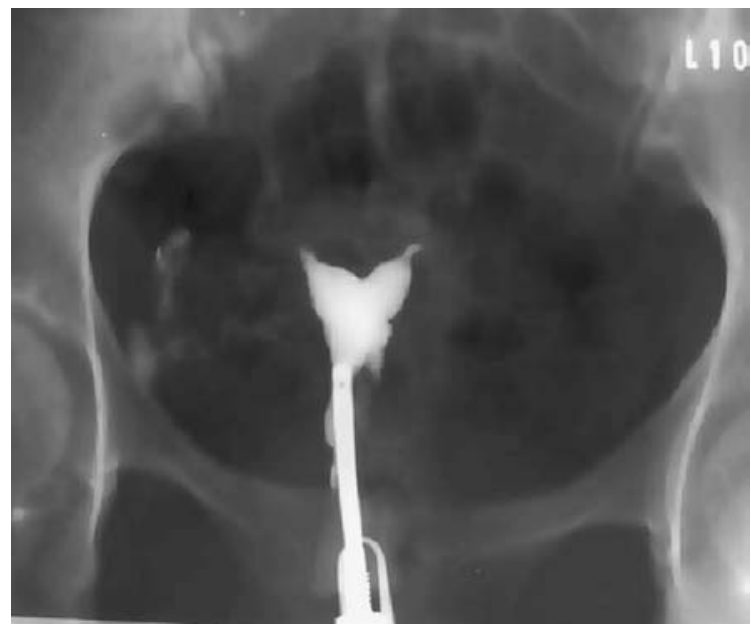

Fig. 10 Reconstruction of the uterine cavity after previous insufficient Strassman metroplasty. Final result after resectoscopy ratio $20 \%$

$5,6,7,8,9,10)$. In preoperative evaluation of the uterine cavity, we used sonohysterography $(n=5)$ and hysteroscopy $(n=10)$. The methods are presented in Fig. 11. Other factors of infertility were excluded before surgery.

In group I $(n=28)$, the ratio of the uterine cavity height and distance of the uterine horns apices was measured and calculated before and after hysteroscopic septum resection. The principle of the ratio calculation is given in Figs. 1 and 2. In groups II and III, the preoperative diagnosis and postoperative assessment of septum resection were not calculated.

According to the aim of our study, we analysed group $\mathrm{I}$, and this group was divided into subgroups according to the calculated ratio: I-A (ratio $<10 \%, n=17$ ); I-B (ratio $>10 \%, n=11$ ).

The pregnancy rate in group I was $82.1 \%(n=23)$ and in groups II and III, 26.7\% $(n=4)$ (significantly different, $P<0.01)$. The pregnancy rate in group I-A was $88.2 \%(n=15)$ and in group I-B, 72.7\% $(n=8)$ (not significantly different, $P>0.01$ ).

The incidence of spontaneous abortion in group IA was $6.7 \%(n=1)$ and in the group I-B, $37.5 \%$ $(n=3)$ (difference, $P<0.1)$. The results and pregnancy rates of all the groups (I, II and III) are shown in Table 1.

\section{Discussion}

Hysteroscopic treatment of congenital uterine malformations is a method of choice in infertile women, achieving high postoperative pregnancy rates $[8,10,16]$. In our study, we observed $27(62,8 \%)$ intrauterine pregnancies, and there were $4(14.8 \%)$ spontaneous abortions among them. No ectopic pregnancy was observed. Interestingly, the difference in pregnancy rates between the studied group I and groups II and III was significantly different. We can only speculate that this statistically proven difference between these groups is connected with different pre- and postoperative methods of uterine cavity evaluation.

The aim of our study was to ascertain anatomical changes of the uterine cavity after hysteroscopic septum resection $[5,12,14]$. We used hysterography in our study, although we know that other imaging methods, such as ultrasonography, are available $[5,10]$. The final results of hysteroscopic septum resection are closely related to the operator's experience $[8,9,15,16]$. The exact knowledge of uterine cavity changes before and after surgery gives interesting information even to a skilled surgeon. Fedele et al. published that a small residual septum $(<1 \mathrm{~cm})$ after hysteroscopic septum resection does not impair the reproductive outcome [5].In our study, we calculated an index where the septum as well as the uterine cavity changes after resection are evaluated. Our results showed a higher incidence of spontaneous abortions when the calculated ratio was $>10 \%$. Especially in difficult cases such as reoperation after failed Strassman metroplasty, hypoplastic uterus or complete septate uterus, the hysteroscopic resection could be connected with incomplete resection. The residual septum could be connected with persistent pregnancy loss.

In our retrospective study we tried to point out interesting anatomical changes of the uterine cavity after hysteroscopic septum resection and reproductive outcome according to ratio of the uterine cavity height and the distance of the uterine horns apices.
Fig. 11 Methods of uterine septum evaluation

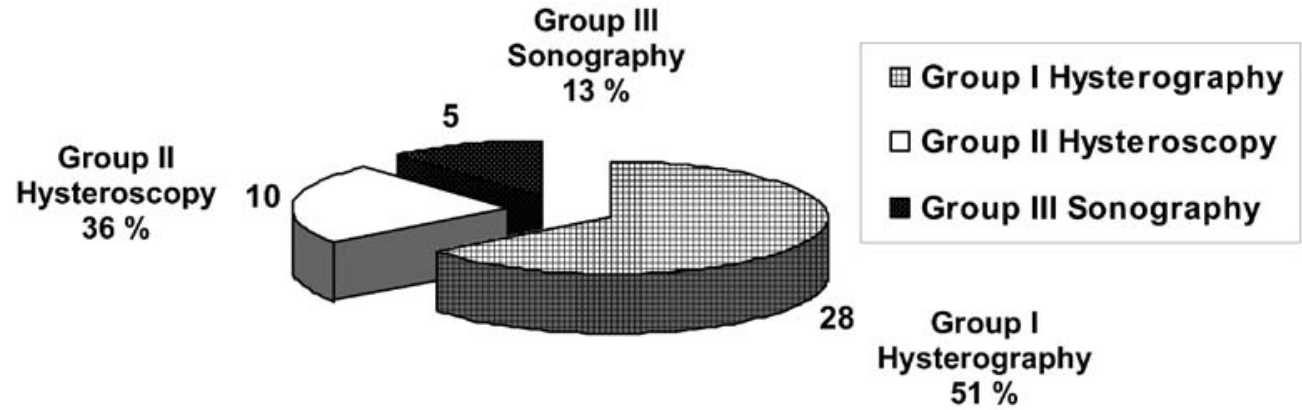

Group III nography 
Table 1 Pre- and postoperative evaluation and pregnancy outcome

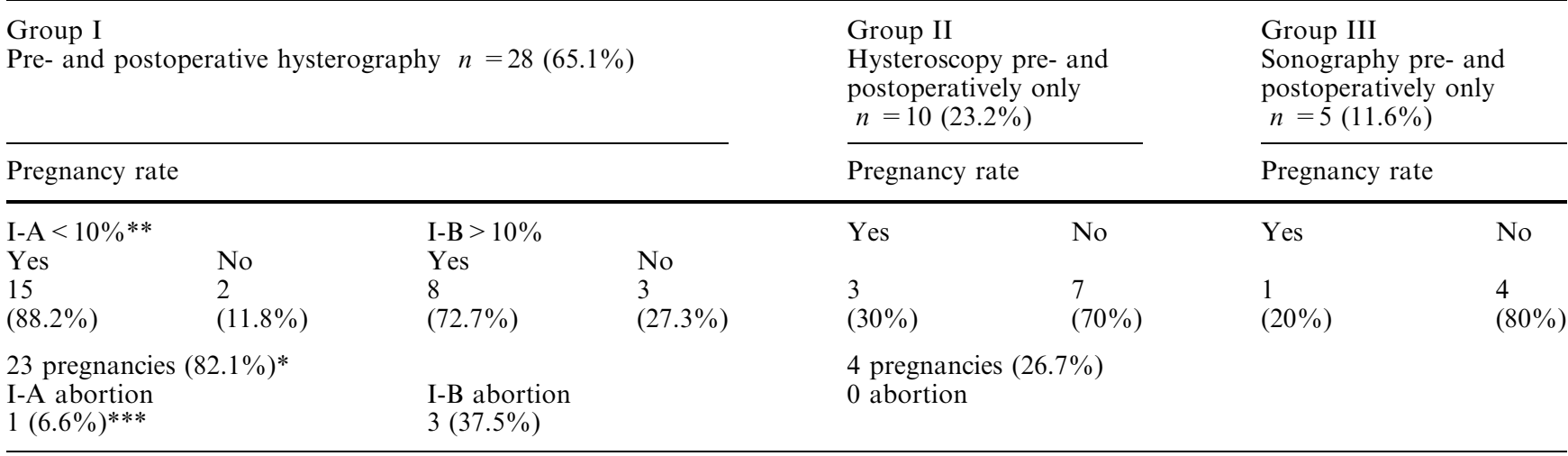

$* P<0.01 ; \mathrm{X}^{2}=12.87, * * P>0.01 ; \mathrm{X}^{2}=1.09, * * * P<0.1 ; \mathrm{X}^{2}=3.4$

The pregnancy rate in the group with index $<10 \%$ was $88.2 \%$ and in the group with residual septum (index $>10 \%$ ), $72.7 \%$ (not significantly different). There was a higher incidence of spontaneous abortions in the group with residual septum $(37.5 \%)$ than in the group with no residual septum $(6.7 \%)$ (different).

Incomplete correction leaving residual uterine septum may influence the uterine capacity negatively. The residual septum could cause a higher incidence of pathologic implantation with following spontaneous abortion. Different authors have found that the septal endometrium showed defective development with less connective tissue, altered pattern of vascularity and more muscle fibers inside the septum $[2,3,6]$. The mechanism by which the septate uterus causes pregnancy loss is not clearly understood [10]. We can only speculate that a higher incidence of spontaneous abortions in group I-B in our study was connected with septal implantation with poor embryo growth. In our opinion, this is a very important fact and has to be discussed with the patient, especially in the above-mentioned difficult cases connected with higher risk of residual septum. Repeated surgical interventions in case of incomplete septum resection are sometimes necessary, but for the patient troublesome. We suppose that our results together with the calculated index will help to understand better the anatomical changes of the uterine cavity.

Reliable diagnosis of the uterine cavity malformations depends on accurate assessment of the uterine cavity. There are several methods available with promising results from three-dimensional ultrasound [10]. In our study, we used hysterography for exact measurement of the uterine cavity changes and index calculation, although we know that this method has only a few indications in gynecology. We hope that the results discussed in our paper will lead to further studies using different imaging methods for precise anatomical evaluation of the uterine cavity.

\section{Conclusion}

When the above-mentioned ratio is $10 \%$ or less, reproductive failure is not expected. There is a higher incidence of spontaneous abortion when the calculated ratio is more than $10 \%$. Therefore, especially in difficult cases of uterine septum resection (e.g., hypoplastic septate uterus, repeat pregnancy loss or failure after surgery), the evaluation of surgical outcome after hysteroscopic septum resection by radiography is still useful.

\section{Reference}

1. Buttram VC (1983) Müllerian anomalies and their management. Fertil Steril 40:159-164

2. Candiani GB, Fedele L, Zamberletti D, De Virgiliis D, Carinelli S (1983) Endometrial patterns in malformed uteri. Acta Eur Fertil 14:35-42

3. Dabirashrafi H, Bahadori M, Mohammed K, Alavi M, Moghadami-Tabrizi N, Zandinejad K, Ghafari V (1995) Septate uterus: new idea on histologic features of the septum in this abnormal uterus. Am J Obstet Gynecol 171:105-107

4. Fedele L (1999) Hysteroscopic metroplasty for Septate uterus (abstract). Eighth Congress of ESGE, Stockholm, p 57

5. Fedele L, Bianchi S, Marchini M, Mezzopane R, Di Nola G, Tozzi L (1996) Residual uterine septum of less than $1 \mathrm{~cm}$ after hysteroscopic metroplasty does not impair reproductive outcome. Hum Reprod 11:727-729

6. Fedele L, Bianchi S, Marchini M, Franchi D, Tozzi L, Dorta M (1996) Ultrastructural aspects of endometrium in infertile women with septate uterus. Fertil Steril 65:750-752

7. Gaucherand P, Piacenza JM, Salle B, Rudigoz RC (1995) Sonohysterography of the uterine cacity: preliminary investigations. J Clin Ultrasound 23:339-348

8. Goldenberg M, Sivan E, Sharabi Z, Mashiach S, Lipitz S, Seidman D (1995) Reproductive outcome following hysteroscopic management of intrauterine septum and adhesions. Hum Reprod 10:2663-2665

9. Grimbizis G, Caus M, Vlasem K, Tournaye H, DeMunck L, Devroey P (1998) Hysteroscopic septum resection in patients with recurrent abortions or infertility. Hum Reprod 13:11881193 
10. Homer HA, Li TC, Cooke D (2000) The septate uterus: a review of management and reproductive outcome. Fertil Steril 73:1-14

11. Loverro G, Nappi L, Vicino M, Carriero C, Vimercati A, Selvaggi L (2001) Uterine cavity assessment in infertile women: comparison of transvaginal sonography and hysteroscopy. Eur J Obstet Gynecol Reprod Biol 100:67-71

12. Ott DJ, Fayez JA (1991) Hysterosalpingography - a text and atlas. Urban and Schwarzenberg, Baltimore, Munich

13. Raga F, Bauset C, Remohi J, Bonilla-Musoles F, Simon C, Pellicer A (1997) Reproductive impact of congenital müllerian anomalies. Hum.Reprod 12:2277-2281
14. Sörensen SS (1983) Fundal contour of the uterine cavity in the new syndrome of minor müllerian anomalies and oligomenorrhea: A prospective controlled study. Am J Obstet Gynecol 145:659-667

15. Venturoli S, Kolombo FM, Vianello F, Seracchioli R, Possati G, Paradisi R (2002) A study of hysteroscopic metroplasty in 141 women with a septate uterus. Arch Gynecol Obstet 266:157-159

16. Xia,E (2003) Experience of managing septate uterus (abstract). XII ${ }^{\text {th }}$ Annual Congress of ISGE, Cancun, $p 60$ 